\title{
EFFECTS OF SHOCK ON HYPERSONIC BOUNDARY LAYER STABILITY
}

\author{
F. Pinna and P. Rambaud \\ von Karman Institute \\ 72 Chaussée de Waterloo, Rhode-St-Genèse 1640, Belgium
}

\begin{abstract}
The design of hypersonic vehicles requires the estimate of the laminar to turbulent transition location for an accurate sizing of the thermal protection system. Linear stability theory is a fast scientific way to study the problem. Recent improvements in computational capabilities allow computing the flow around a full vehicle instead of using only simplified boundary layer equations. In this paper, the effect of the shock is studied on a mean flow provided by steady Computational Fluid Dynamics (CFD) computations and simplified boundary layer calculations.
\end{abstract}

\section{INTRODUCTION}

The renewed interest in the design of hypersonic vehicles is encouraging the transition community to study high speed flows. A common practice in the design of such a vehicle is the assumption of a fully turbulent boundary layer leading to a conservative sizing of the thermal protection system (TPS). Including the effect of turbulence makes the solution of the flow inherently more complex and stiff, especially when chemical reactions are also considered. The result is a conservative estimate of the heat flux on the wall implying a heavier vehicle which is far from the optimal design. Thermal protection system has a considerable impact on the performance of a new vehicle mainly due to its weight, so that a lighter layer of it would be highly desirable. On the other hand, without this conservative approach, poor modeling could result in overheating of the whole structure, damaging the TPS and eventually causing the loss of the vehicle and the failure of the mission. Moreover, in the case of laminar to turbulent transition, an overshoot of the heat flux with respect to a turbulent prediction is likely to appear, making the turbulent case not the worst possible one which requires a higher safety margin. It is not a coincidence that the hypersonic community has started investigating the transition modeling with increasing interest in order to decrease the overall uncertainty related to the heat flux prediction on a hypersonic vehicles. 
One of the main features of a hypersonic flow is the presence of a strong shock in front of the body. This has several implications on the flow field and consequently on the boundary layer stability. The most apparent effect is the high temperature behind the shock which could rise up to few thousand Kelvins. Molecular oxygen and nitrogen dissociate around 2000 and $4000 \mathrm{~K}$, respectively.

This is an important effect to be taken into account in stability computations; one of the first analyses focusing on reacting flows was published by Malik and Anderson [1] on a flat plate at Mach 10 and 15. The main consequence, as expected, was the mean profile shape modification, and this difference alone would be enough to modify the growth rate of the disturbances. Air properties considered as a mixture of dissociated gas, were taken into account in their linear stability model as well as in the mean flow, showing the overall effect on the flow stability. It is worth noting that in contribution by Malik and Anderson [1], a self-similar profile was used and, therefore, effects caused by the leading edge shape (such as the entropy layer) were not taken into account. It is clear that for these conditions, air should be considered as a mixture of gases and reactions among different species should also be taken into account.

Another feature of hypersonic flows is the presence of an entropy layer [2] if a blunt nosed body is used. The flow is remarkably different from a low-supersonic compressible flow because of the large entropy gradients introduced by the nose itself.

Shock waves also act directly on the flow stability as they react to upwind perturbations modifying the so called receptivity problem [3]. It is known that any kind of wave interacting with the shock boundary generates all three different types of modes, which are the acoustic mode, the vorticity mode and the entropy mode [4-7]. Even when the way perturbations penetrate through the shock layer is not considered, the fact that the shock vibrates with a certain frequency affects the stability of the boundary layer. This aspect was addressed, probably for the first time, by Petrov [8], who used a linearized Rankine-Hugoniot $(\mathrm{R}-\mathrm{H})$ relation as the shock boundary condition. Cowley and Hall [9] used the triple deck theory with also an $\mathrm{R}-\mathrm{H}$ boundary condition. Chang et al. [10] used the unsteady $\mathrm{R}-\mathrm{H}$ and a similar approach was proposed also by Herbert and Esfahanian [11]. Stuckert [12] formulated a slightly different approach, although starting from unsteady R-H. In many cases, approximated self-similar boundary layer profiles were used and cropped at the shock location as predicted by a simple Euler computation.

This approach is efficient, yet, it neglects the shock boundary layer interactions deforming the mean flow profiles and slightly displacing the shock away from the wall.

The work presented studies the flow stability on a wedge alternatively evaluated by means of a CFD solver or a self-similar profile. Chemical reactions are taken into account considering the flow in chemical equilibrium on a simple 
wedge geometry. The effect induced by the nose bluntness is not analyzed in this paper as the wedge is assumed to have a perfectly sharp leading edge.

\section{GOVERNING EQUATIONS}

\subsection{Mean Flow}

As the temperature increases, the fluid departs from the ideal behavior of a calorically perfect gas. When the temperature is high enough for the molecule to dissociate, air should be considered as a mixture of $n_{s}$ species, whose composition depends on the flow conditions and on the kind of modeling included in the computation. The flow could be considered in chemical equilibrium, chemical nonequilibrium, or thermochemical nonequilibrium.

Dalton's law on partial pressure can be used to retrieve the pressure of the mixture, which is then given by

$$
p=\mathcal{R} \sum_{i=1}^{n_{s}} \frac{y_{i}}{M_{i}} \rho T
$$

where $\mathcal{R}, y_{i}$, and $M_{i}$ are respectively the universal gas constant, the mass fraction and the molecular weight of the $i$ th species. Other variables, such as enthalpy, are expressed in a similar fashion:

$$
h=\sum_{i=1}^{n_{s}} \frac{\rho_{i} h_{i}}{\rho} .
$$

These quantities are proportional to the mass fraction of each species and, therefore, the computation of other properties requires the definition of the mixture. In chemical equilibrium, the mixture composition can be evaluated once the pressure, the temperature, and the elemental mole fraction are given $\left(79 \% \mathrm{~N}_{2}\right.$ and $21 \% \mathrm{O}_{2}$ in the presented case). Here, the pressure $p$ and the temperature $T$ are chosen, but other state variables such as internal energy, entropy, density could be used as well. Navier-Stokes equations remain formally unchanged:

$$
\begin{aligned}
\frac{\partial \rho}{\partial t}+\nabla \cdot(\rho \underline{u}) & =0 ; \\
\rho \frac{\partial \underline{u}}{\partial t} & =-\nabla p+\frac{1}{\operatorname{Re}} \nabla \cdot(\underline{\underline{\tau}}) ; \\
\rho \frac{D h}{D t} & =E c \frac{D p}{D t}+\frac{E c}{\operatorname{Re}}(\nabla \cdot(\underline{\underline{\tau}} \underline{u})-\underline{u} \nabla \cdot \underline{\tau})+\frac{1}{\operatorname{Re} \cdot \operatorname{Pr}} \nabla \cdot \underline{q}
\end{aligned}
$$


where $\underline{u}$ represents the velocity vector; $\underline{q}=k \nabla T$ is the heat flux; and $\underline{\tau}$ $=\lambda(\nabla \cdot \underline{u}) \underline{\underline{I}}+\mu\left(\nabla \underline{u}+\nabla \underline{u}^{\mathrm{T}}\right)$ is the shear stress tensor, with $k, \lambda$, and $\mu$ representing the thermal conductivity, the second and the first coefficients of viscosity, respectively. The enthalpy $h$ and the internal energy $e$ in the energy equation cannot be further simplified into a product of a specific heat constant (pressure or volume) and temperature so the terms containing, for instance, the enthalpy $h(p, T)$ are written as follows:

$$
\frac{\partial h}{\partial t}=\left.\frac{\partial h}{\partial p}\right|_{T} \frac{d p}{d t}+\left.\frac{\partial h}{\partial T}\right|_{p} \frac{d T}{d t} .
$$

The mean flow is provided by means of CFD and boundary layer computations. The CFD computations are performed using the steady Finite Volume solver in COOLFLUID [13] for the chemically reacting flow, and CFD++ commercial package for the perfect gas calculations. A self-similar boundary layer is retrieved by means of a custom code using a 4th order Runge-Kutta shooting method for both a calorically perfect gas and a Local Thermodynamic Equilibrium (LTE) flows.

For a self-similar profile on a flat plate with no pressure gradients, one can write:

$$
\begin{aligned}
\left(c f^{\prime \prime}\right)+f f^{\prime \prime} & =0 \\
\left(a_{1} g^{\prime}+a_{2} f^{\prime} f^{\prime \prime}\right)^{\prime}+f g^{\prime} & =0
\end{aligned}
$$

where

$$
\begin{gathered}
f^{\prime}=\frac{u}{u_{e}} ; \quad c=\frac{\rho \mu}{\rho_{e} \mu_{e}} ; \quad g=\frac{H}{H_{e}} ; \\
a_{1}=\frac{c}{\operatorname{Pr}} ; \quad a_{2}=\frac{U_{e}^{2}}{H_{e}}\left(1-\frac{1}{\operatorname{Pr}}\right) c .
\end{gathered}
$$

It is worth noting that the use of LTE hypothesis allows finding a self-similar profile; this would not be possible considering chemical and thermochemical nonequilibria.

\subsection{Linear Stability Theory}

Linear Stability Theory (LST) computations are performed using the VESTA toolkit developed at the von Karman Institute [14] on the mean flow profile provided by the aforementioned solvers. The LST equations for the compressible case are retrieved by splitting the instantaneous flow into a time averaged flow field and a perturbation field:

$$
Q(x, y, z, t)=\bar{Q}(x, y, z)+q^{\prime}(x, y, z, t)
$$


where $q^{\prime}$ represents a generic variable disturbance. Equation (1) is substituted in the Navier-Stokes equations and the result is linearized around the mean flow. A more detailed discussion could be found in $[15,16]$.

A difference between the simple compressible LST code and the one for flows with chemical reactions in equilibrium lies in the decomposition of the transport properties: for a calorically perfect gas they depend only on temperature, while for LTE flows in general, they are a function of two thermodynamic variables. As $p$ and $T$ are chosen, a generic transport property is written as follows:

$$
\mu^{\prime}=\frac{d \mu}{d T} T^{\prime}+\frac{d \mu}{d p} p^{\prime}
$$

Another key hypothesis assumes that each perturbation propagates independently. These are called modes and for a general disturbance are represented by

$$
q^{\prime}(x, y, z, t)=\tilde{q} \exp (i \alpha x+i \beta z-i \omega t)+c . c .
$$

where the $\tilde{q}$ term is the amplitude function; $\alpha$ and $\beta$ are the streamwise and spanwise wavenumbers; and $\omega$ is the frequency.

For LTE cases, an air-5 mixture $\left(\mathrm{N}_{2}, \mathrm{O}_{2}, \mathrm{~N}, \mathrm{O}\right.$, and $\left.\mathrm{NO}\right)$ is used to compute the rates and the flow properties, and the gas state equation is rewritten as

$$
p=\rho r T \zeta
$$

where $r$ is the gas constant for the undissociated gas $\left(\mathrm{N}_{2}-\mathrm{O}_{2}\right.$ mixture) and $\zeta$ $=M_{\text {und }} / M_{\text {dis }}$ is the compressibility factor.

The numerical method used is Chebyshev collocation and it is similar to the one proposed in [16]. The general approach for LTE flows is the same that in [14].

The reference quantities $l=\sqrt{\nu_{e} x_{e} / U_{e}}, U_{e}, \rho_{e}, \rho_{e} U_{e}^{2}, l / U_{e}$, and $T_{e}$ are used to nondimensionalize the length, velocity, density, time, and the temperature in the Navier-Stokes equations. The reference viscosity (both first and second coefficients) corresponds to the value computed for the external temperature $T_{e}$ and the pressure $p_{e}$.

The set of equations for linear stability of an LTE flow are:

\section{continuity equation}

$$
H P\left(-\frac{T_{y} \zeta_{T}}{\zeta}-\frac{T_{y}}{T}\right) \tilde{v}+F H \Psi \tilde{p}+G H \frac{P}{T} \Psi \tilde{T}+i \beta H P \tilde{w}+H P \tilde{v}_{y}+i \alpha H P \tilde{u}=0 ;
$$




\section{$x$ momentum equation}

$$
\begin{array}{r}
\frac{H P}{T \zeta}\left(\Psi \tilde{u}+U_{y} \tilde{v}\right)=\frac{1}{\operatorname{Re}}\left(\mu_{P} U_{y y}+\mu_{P T} T_{y} U_{y}-i \alpha\right) \tilde{p}+\frac{1}{\operatorname{Re}}\left(\mu_{T} U_{y y}+\mu_{T T} T_{y} U_{y}\right) \tilde{T} \\
+\frac{1}{\operatorname{Re}}\left[\mu_{p} U_{y} \tilde{p}_{y}+\mu_{T} U_{y} \tilde{T}_{y}+\left(i \alpha \mu_{T} T_{y}\right) \tilde{v}+\mu_{T} T_{y} \tilde{u}_{y}+i \alpha(\mu+\lambda) \tilde{v}_{y}\right] \\
-\frac{1}{\operatorname{Re}}\left[\alpha \beta(\mu+\lambda) \tilde{w}+u_{y y} \mu-\left(\alpha^{2}(\lambda+2 \mu)+\mu \beta^{2}\right) \tilde{u}\right]
\end{array}
$$

\section{$y$ momentum equation}

$$
\begin{array}{r}
\frac{H P}{T \zeta} \Psi \tilde{v}=\frac{1}{\operatorname{Re}}\left[\left(i \beta \mu_{P} W_{y}+i \alpha \mu_{P} U_{y}\right) \tilde{p}+\left(i \beta \mu_{T} W_{y}+i \alpha \mu_{T} U_{y}\right) \tilde{T}\right] \\
+\frac{1}{\operatorname{Re}}\left[\left(2 \mu_{T}+\lambda_{T}\right) T_{y} \tilde{v}_{y}+i \beta \lambda_{T} T_{y} \tilde{w}+i \alpha \lambda_{T} T_{y} \tilde{u}+i \beta(\mu+\lambda) \tilde{w}_{y}\right]-\tilde{p}_{y} \\
+\frac{1}{\operatorname{Re}}\left[(2 \mu+\lambda) \tilde{v}_{y} y-\mu\left(\beta^{2}+\alpha^{2}\right)+i \alpha(\mu+\lambda) \tilde{u}_{y}\right]
\end{array}
$$

\section{$z$ momentum equation}

$$
\begin{aligned}
& \frac{H P}{T \zeta}\left(\Psi \tilde{w}+W_{y} \tilde{v}\right)=\frac{1}{\operatorname{Re}}\left[\left(\mu_{P} W_{y y} \mu_{P T} T_{y} W_{y}\right) \tilde{p}\right. \\
& \left.+\left(\mu_{T} W_{y y}+\mu_{T} T T_{y} W_{y}\right) \tilde{T}+\mu_{P} W_{y} \tilde{p}_{y}\right] \\
& +\frac{1}{\operatorname{Re}}\left[\mu_{T} W_{y} \tilde{T}_{y}+\mu_{T} T_{y} \tilde{w}_{y}+i \beta \mu_{T} T_{y}+\mu \tilde{w}_{y y}\right]-i \beta \tilde{p} \\
& -\frac{1}{\operatorname{Re}}\left[\left(\beta^{2}(2 \mu+\lambda)+\alpha^{2} \mu\right) \tilde{w}+i \beta \mu \tilde{v}_{y}-\alpha \beta \mu \tilde{u}+i \beta \lambda \tilde{v}_{y}-\alpha \beta \lambda \tilde{u}\right] ;
\end{aligned}
$$

energy equation

$$
\begin{gathered}
\frac{H P}{T \zeta}\left[\left(h_{T} \Psi \tilde{T}+h_{P} \Psi \tilde{p}\right)+h_{T} T_{y} \tilde{v}\right]=\frac{1}{\operatorname{Pr} \cdot \operatorname{Re}}\left[\left(2 k_{T} T_{y}\right) \tilde{T}_{y}+k_{P} T_{y} \tilde{p}_{y}+k \tilde{T}_{y y}\right] \\
+\frac{E c}{\operatorname{Re}}\left(\left(W_{y}^{2}+U_{y}^{2}\right)\left(\mu_{P} \tilde{p}+\mu_{T} \tilde{T}\right)\right. \\
\left.+i \cdot 2 \mu\left(\beta W_{y}+\alpha U_{y}\right) \tilde{v}+2 \mu W_{y} \tilde{w}_{y}+2 \mu U_{y} \tilde{u}_{y}\right)+E c \Psi \tilde{p} \\
+\frac{1}{\operatorname{Pr} \operatorname{Re}}\left[\left(k_{T} T_{y y}^{2}+k_{T T} T_{y}^{2}-\left(\alpha^{2}+\beta^{2}\right) k\right) \tilde{T}+\left(k_{P} T_{y y}+k_{P T} T_{y}^{2}\right) \tilde{p}\right]
\end{gathered}
$$


where $\Psi=i \alpha U+i \beta W-i \omega, F=1-\left.(\partial \ln \zeta / \partial p)\right|_{T}, G=1+\left.(\partial \ln \zeta / \partial T)\right|_{p}$, and $H=c_{p} E c / r$. This set of equations could be reduced to a system in the form:

$$
\left[A D^{2}+B D+C\right] \chi=0
$$

where $\chi=[\tilde{u}, \tilde{v}, \tilde{p}, \tilde{T}, \tilde{w}]$ is the unkonwn vector and $D=d / d y$ is the derivative operator.

When the presence of the shock is neglected, one can assume that perturbations vanish at the wall and in the far field giving

$$
\left.[\tilde{u}, \tilde{v}, \tilde{T}, \tilde{w}]\right|_{\text {wall }} ^{\infty}=0 .
$$

The boundary condition on the pressure is treated differently as it is impossible to directly impose a value. Therefore, the $y$-momentum equation is evaluated at the boundaries, providing the needed boundary conditions.

\subsection{Shock as a Boundary Condition}

In case the shock is sufficiently close to the boundary layer, perturbations do not vanish in the far field but interact with the shock itself. For this reason, the standard boundary condition cannot be applied anymore. Following the development of $[10,17]$, a set of boundary conditions was implemented representing the role of the shock in the boundary layer stability. This set of equations comes from a linearization of the unsteady Rankine-Hugoniot jump relations that could be generally written as:

$$
\frac{\partial f}{\partial t}[\mathbf{Q}]+\frac{\partial f}{\partial x}[\mathbf{E}]-[\mathbf{F}]+\frac{\partial f}{\partial z}[\mathbf{G}]=0
$$

where

$$
\begin{aligned}
& \mathbf{Q}=[\rho, \rho u, \rho v, \rho w, e]^{\mathrm{tr}} ; \\
& \mathbf{E}=\left[\rho u, \rho u^{2}, \rho u v, \rho u w,(e+p) u\right]^{\mathrm{tr}} ; \\
& \mathbf{F}=\left[\rho v, \rho u v, \rho v^{2}, \rho v w,(e+p) v\right]^{\mathrm{tr}} ; \\
& \mathbf{G}=\left[\rho w, \rho u w, \rho v w, \rho w^{2},(e+p) w\right]^{\mathrm{tr}}
\end{aligned}
$$

Rankine-Hugoniot equations are linearized with respect to the mean shock position $\bar{y}_{s}=f(x, y, z)$, and by introducing harmonic waves, one gets:

$$
i(\alpha[\overline{\mathbf{E}}]+\beta[\mathbf{G}]-\omega[\mathbf{Q}])+\alpha[\tilde{\mathbf{E}}]-[\tilde{\mathbf{F}}]=0
$$


where

$$
\begin{gathered}
\tilde{\mathbf{E}}=\left[\begin{array}{c}
\rho_{2} \tilde{u}+\tilde{\rho} u_{2} \\
\tilde{\rho} u_{2}^{2}+2 \rho_{2} u_{2} \tilde{u}+\tilde{p} \\
\tilde{\rho} u_{2} v_{2}+\rho_{2} v_{2} \tilde{u}+\rho_{2} u_{2} \tilde{v} \\
\tilde{\rho} u_{2} w_{2}+\rho_{2} \tilde{u} w_{2}+\rho_{2} u_{2} \tilde{w} \\
\left(e_{2}+p_{2}\right) \tilde{u}+u_{2}(\tilde{e}+\tilde{p})
\end{array}\right] ; \quad \tilde{\mathbf{F}}=\left[\begin{array}{c}
\rho_{2} \tilde{v}+\tilde{\rho} v_{2} \\
\tilde{\rho} u_{2} v_{2}+\rho_{2} v_{2} \tilde{u}+\rho_{2} u_{2} \tilde{v} \\
\tilde{\rho} v_{2}^{2}+2 \rho_{2} v_{2} \tilde{v}+\tilde{p} \\
\tilde{\rho} v_{2} w_{2}+\rho_{2} \tilde{v} w_{2}+\rho_{2} v_{2} \tilde{w} \\
\left(e_{2}+p_{2}\right) \tilde{v}+v_{2}(\tilde{e}+\tilde{p})
\end{array}\right] ; \\
{[\overline{\mathbf{Q}}]=\bar{Q}_{1}-\bar{Q}_{2} ;[\overline{\mathbf{E}}]=\bar{E}_{1}-\bar{E}_{2} ;[\overline{\mathbf{G}}]=\bar{G}_{1}-\bar{G}_{2} .}
\end{gathered}
$$

These equations are in dimensional form, which is valid for both CPG (calorically perfect gas) and LTE flows. This happens because there is no formal change in the way the governing equations are written. Nevertheless, the actual implementation is nondimensional, making the two cases quite different. Skipping the details of the derivation one should have in mind that nondimensional numbers appear only in the jump relation associated to the energy equation. When, for instance, one refers to the mean internal energy it should be considered as

$$
e_{2}=\rho_{2} \epsilon_{2}+\gamma E c\left(\frac{u_{2}^{2}+v_{2}^{2}+w_{2}^{2}}{2}\right)
$$

where $\epsilon$ is the specific internal energy and the subscript "2" stands for the quantity behind the shock. The perturbation of this quantity will be:

$$
e_{2}^{\prime}=\rho_{2}^{\prime} e_{2}+\rho_{2} e_{2}^{\prime}+\gamma E c\left(\rho_{2}^{\prime}\left(\frac{u_{2}^{2}+v_{2}^{2}+w_{2}^{2}}{2}\right)+\rho_{2}\left(u_{2} u_{2}^{\prime}+v_{2} v_{2}^{\prime}+w_{2} w_{2}^{\prime}\right)\right) \text {. }
$$

\section{RESULTS}

Wedges present a good test case as they produce a relatively simple flow field, they are two-dimensional, and, therefore, fast to compute, and, besides, a selfsimilar boundary layer solution can be found. The first test is the one presented by Esfahanian [17] where a Mach 8 flow with an adiabatic wall is computed on a wedge with a half angle of $5^{\circ}$. It should be noted that the actual self-similar profile is computed with the condition behind the shock that is $M=6.86$ and $T=71.88 \mathrm{~K}$ while the wall is adiabatic. This computation is intended for the verification of the computer code. In Fig. $1 a$, the case for $\mathrm{Re}=590.91$ shows a similar behavior for the two computations highlighting the stabilizing role of the shock at low frequencies. Minor differences could be found near the minimum of the shock boundary condition growth rate; nevertheless, the computation is satisfactorily close to the one presented in [17].

The same comparison is carried out for a different Reynolds at the same condition. At $\operatorname{Re}=1557.77$, the influence of the shock is lower, and the implementation matches perfectly with the reference solution of Esfahanian. Only the 


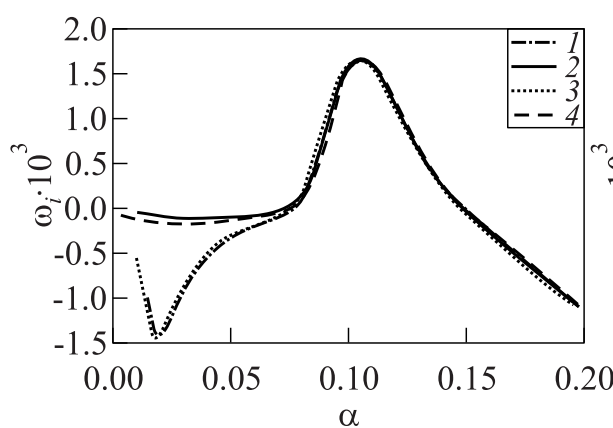

(a)

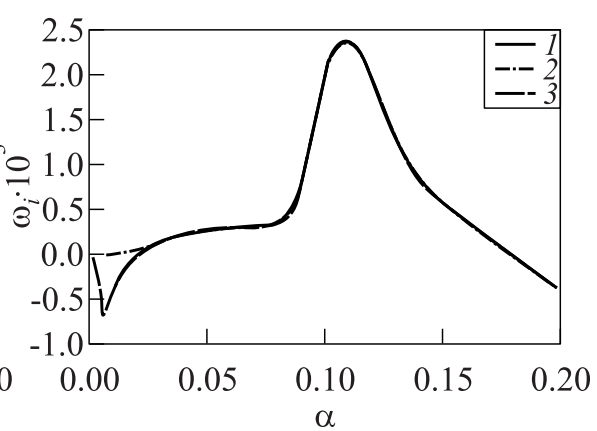

(b)

Figure 1 Growth rate for a Mach 8 flow on a wedge at different boundary conditions (1- shock; 2 - asymptotic; 3 - Esfahanian shock; and 4-Esfahanian asymptotic) and Reynolds numbers: $(a) \operatorname{Re}=590.91$; and $(b) \operatorname{Re}=1557.77$

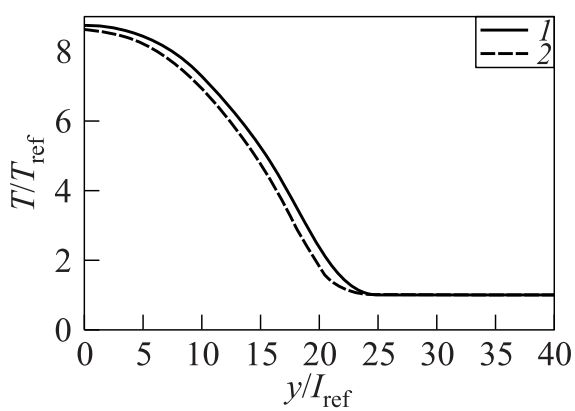

(a)

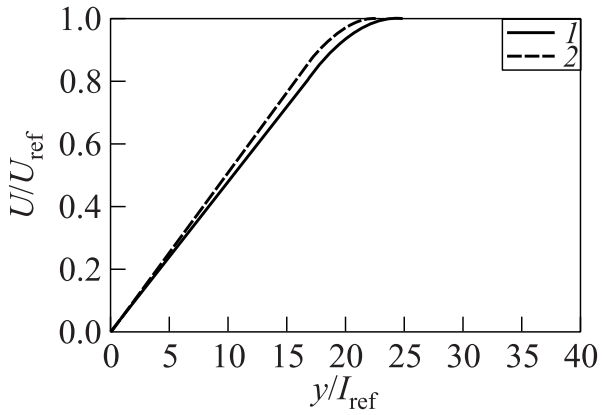

(b)

Figure 2 Nondimensional velocity $(a)$ and temperature $(b)$ profiles for a Mach 8 flow at $\mathrm{Re}=1557.77: 1-\mathrm{CPG}$; and $2-\mathrm{LTE}$

second mode is analyzed as it is the most amplified disturbance in the boundary layer. Oblique first mode waves in this condition have a maximum growth which is far lower than the second mode ones. The LTE shock boundary condition was assessed against this case, expecting a similar result with the one given in Fig. 1, though the boundary layer profile in LTE and CPG slightly differ from each other, as clearly seen in Fig. 2.

Similar behavior of the growth rate is observed in Fig. 3 . The small difference in the mean velocity and temperature profiles leads to a slightly higher second mode peak and a shift in the wavenumber. This phenomenon was already shown in [14]. Interesting enough, the wavenumber shift is less evident when the shock 


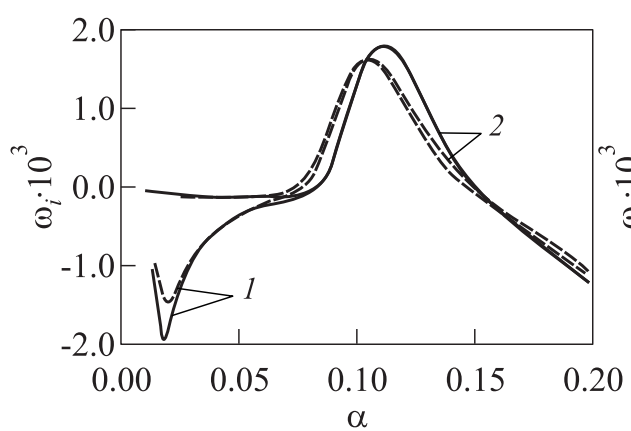

(a)

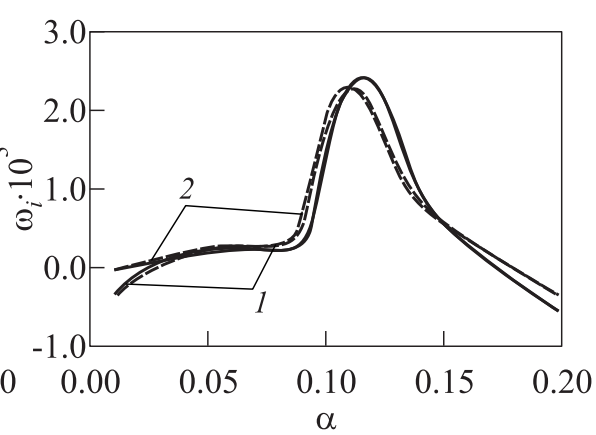

(b)

Figure 3 Growth rate for a Mach 8 flow in CPG (dashed curves) or LTE (solid curves) at $\operatorname{Re}=590.91(a)$ and $\operatorname{Re}=1557.77(b): 1$ - shock boundary conditions; and 2 - asymptotic boundary conditions

boundary condition takes place though a difference in the peak amplitude is still clearly visible in Fig. $3 a$.

It is reasonable to think that the flow computed by means of a full NavierStokes code is different from a self-similar one. Not many publications, at least within the knowledge of the authors, have quantified the error introduced by this assumption. In Fig. 4, such a difference is shown and the CFD profile results are more stable than the self-similar one.

It should be noted that despite the fact that in a Navier-Stokes computation the shock moves further away from the wall with respect to a simple Euler cal-

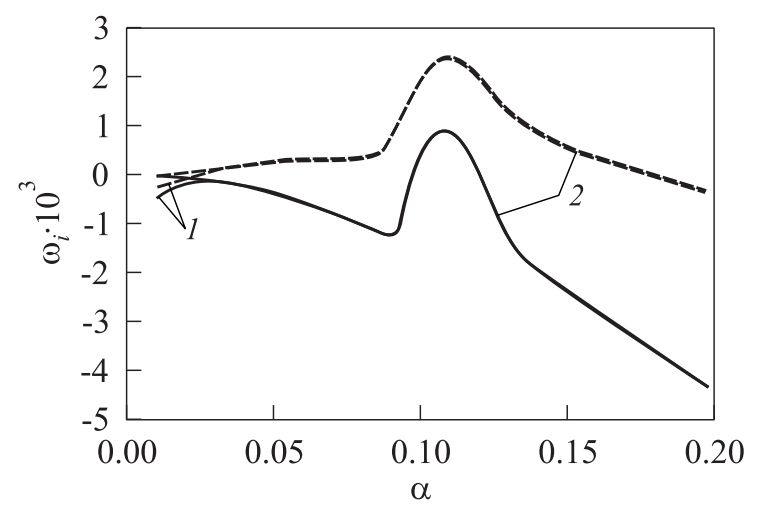

Figure 4 Comparison of self-similar boundary layer flow (dashed curves) against full Navier-Stokes solution (solid curves) at $\operatorname{Re}=1557.77$ and $\mathrm{M}=8: 1$ - shock boundary conditions; and 2 - asymptotic boundary conditions 


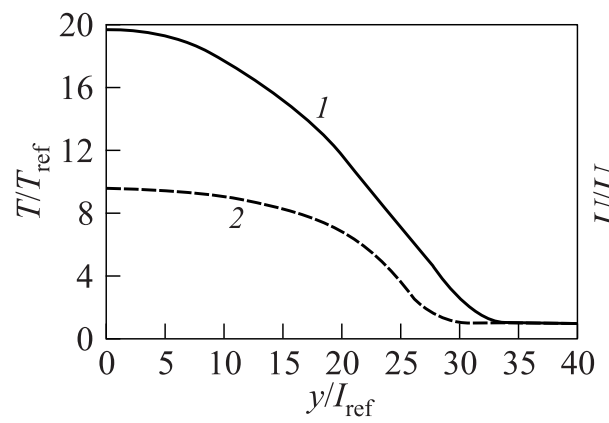

(a)

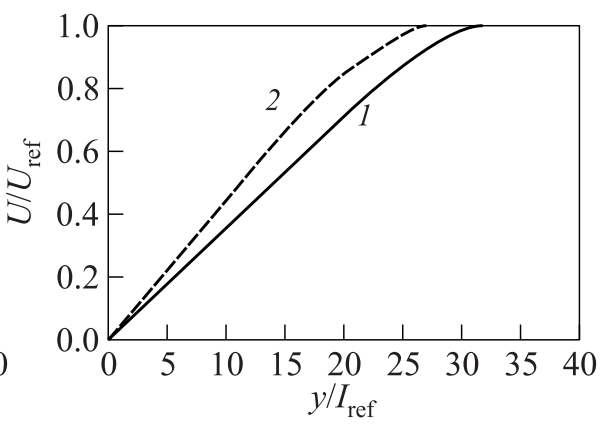

(b)

Figure 5 Nondimensional velocity $(a)$ and temperature $(b)$ profiles for a Mach 14 flow $\mathrm{Re}=1557.77: 1-\mathrm{CPG}$; and 2 - LTE

culation, the wave number at which the shock boundary condition departs from the asymptotic one is not sensibly different. It is also evident that considering a downstream location on the wedge, i. e., increasing the Reynolds number, the shock gets farther away from the wall, affecting less the boundary layer. Also, while increasing the shock distance from the wall, the wavelength affected by the shock progressively increases. This is true regardless of the kind of computation done (CFD or self-similar profile).

Nevertheless, the use of similar profile instead of a full Navier-Stokes solution overestimates the perturbation growth which is very different from one case to another.

The second case at higher Mach number was investigated on the same wedge with the assumption that the inlet flow at Mach 14 corresponds to an after shock Mach of 10.755. The temperature and the pressure behind the shock are $350 \mathrm{~K}$ and $17502.45 \mathrm{~Pa}$, respectively, and the wall is adiabatic as in the previous case.

One immediately understands that the shock height is strictly related not only to Reynolds number, but, for a given geometry, to Mach number as well. With the increase of Mach number, the shock becomes shallower so that the shock boundary condition is potentially more useful. With these conditions, the difference between the CPG mean flow and the LTE one is remarkable, as seen in Fig. 5. The LTE flow has a thinner boundary layer and its maximum temperature at the wall is half the one of the CPG. In order to understand the effects of the Mach number, the shock is kept approximately at the same distance from the wall. For this reason, at Mach 14 the case at $\mathrm{Re}=3760$ is introduced and the profile at $\operatorname{Re}=590.91$ is discarded.

As one would expect, the heavy difference in mean profile affects the growth rate and this is evident in Fig. $6 b$ and Fig. $6 a$. The LTE flow shows two peaks 


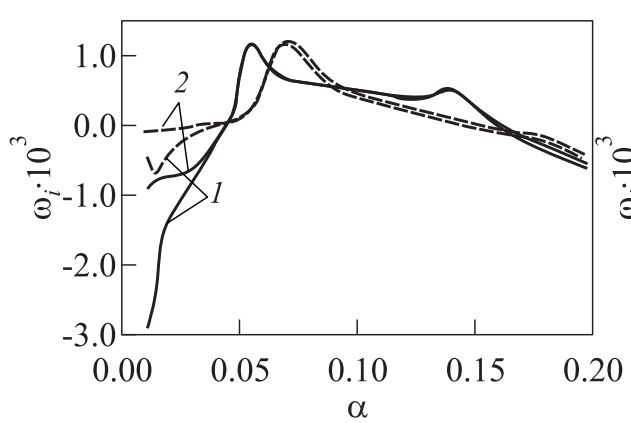

(a)

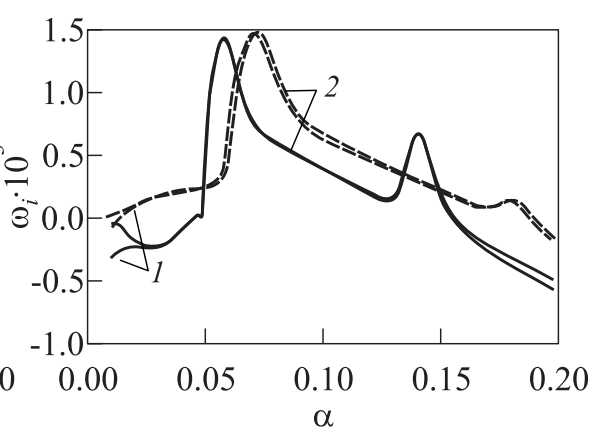

(b)

Figure 6 Growth rate for a Mach 14 flow in CPG (dashed curves) and LTE (solid curves) at $\operatorname{Re}=1557.77(a)$ and $3760(b): 1$ - shock boundary conditions; and 2 asymptotic boundary conditions

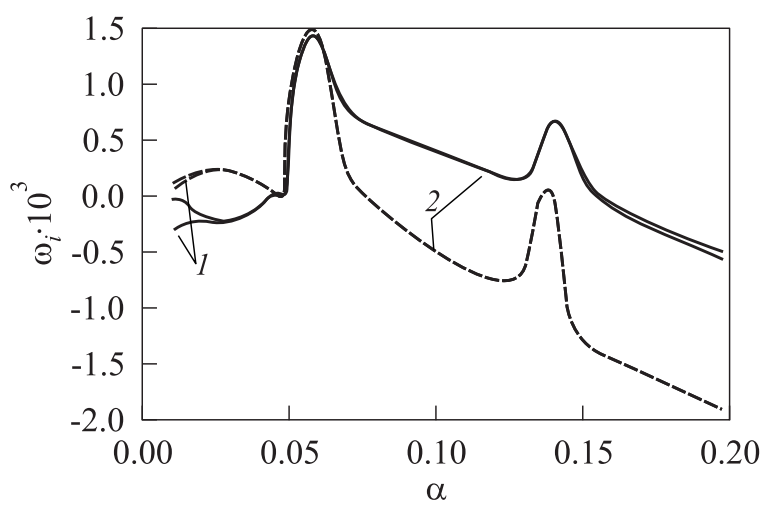

Figure 7 Comparison of self-similar boundary layer flow (dashed curves) against full Navier-Stokes solution (solid curves) for a Mach 14 flow at $\mathrm{Re}=3760$ : 1 - shock boundary conditions; and 2 - asymptotic boundary conditions

due to the second and third mode and a shift in the wave number. The shock in these cases does not behave qualitatively different from the previous case, but only the quantitative value of the growth rate is different.

The CFD computation and the self-similar profile (Fig. 7) are very close to each other in terms of the first peak, while at higher wavenumbers, the selfsimilar boundary layer becomes progressively more stable. The effect of the shock boundary condition is limited as the shock is pretty far from the wall (shock position to displacement thickness ratio is $y_{s} / \delta^{*} \approx 8$. 


\section{CONCLUDING REMARKS}

The VESTA toolkit has been extended to investigate the influence of the shock on the stability of hypersonic boundary layers. Several self-similar profiles have been computed for CPG and LTE flows in order to study the stability of the boundary layer including the presence of chemical reactions and shocks. This is a typical situation in hypersonics, and the VESTA toolkit is now able to deal with these cases.

It has been found that the shock boundary condition is not particularly affected by the chemical reactions and it behaves similarly to the calorically perfect gas case. Nevertheless, as the energy of the flow increases, the differences between the asymptotic and the shock boundary condition becomes more prominent for LTE than it is for CPG.

On the other hand, the shock has a major effect on the mean flow. The simplified self-similar flow is not able to correctly reproduce the flow in the area of interest thus introducing big errors especially at higher wave numbers.

\section{ACKNOWLEDGMENTS}

The authors would like to acknowledge Prof. Thierry Magin for providing the MUTATION library and Dr. Andrea Lani for the COOLFLUID support.

\section{REFERENCES}

1. Malik, M. R., and E. C. Anderson. 1991. Real gas effects on hypersonic boundarylayer stability. Phys. Fluids A 3(5):803-20.

2. Anderson, J. D. 2000. Hypersonic and high temperature gas dynamics. AIAA.

3. Hu, S., and X. Zhong. 1997. Linear stability of hypersonic flow over a parabolic leading edge. 28th Fluid Dynamics Conference. Snowmass Village, CO.

4. Ribner, H.S. 1954. Convection of a pattern of vorticity through a shock wave. NACA Report No. 1164.

5. McKenzie, J. F., and K. O. Westphal. 1968. Interaction of linear waves with oblique shock waves. Phys. Fluids 11(11):2350-62.

6. Balakumar, P. 2009. Stability of supersonic boundary layers on a cone at an angle of attack. 39th Fluid Dynamics Conference and Exhibit. San Antonio, Texas: AIAA.

7. Fedorov, A. 2011. Transition and stability of high-speed boundary layers. Ann. Rev. Fluid Mech. 43(1):79-95.

8. Petrov, G. V. 1984. Stability of a thin viscous layer on a wedge in hypersonic flow of a perfect gas. In: Laminar-turbulent transition: 2nd IUTAM Symposium Proceedings. Ed. V. V. Kozlov. Springer. 
9. Cowley, S., and P. Hall. 1988. On the stability of hypersonic flow past a wedge. ICASE Technical Report.

10. Chang, C.-L., M. R. Malik, and M. Y. Hussain. 1990. Effects of shock on the stability of hypersonic boundary layers. AIAA Paper No. 90-1448.

11. Herbert, T., and V. Esfahanian. 1993. Stability of hypersonic flow over a blunt body. In: AGARD-CP514 - Theoretical and Experimental Methods in Hypersonic Flows. AGARD.

12. Stuckert, G. K. 1988. Linear stability theory of hypersonic chemically reacting viscous flow. Arizona State University.

13. Lani, A. 2009. An object oriented and high performance platform for aerothermodynamics simulation. Von Karman Institute for Fluid Dynamics.

14. Pinna, F., K. Bensassi, P. Rambaud, O. Chazot, A. Lani, and O. Marxen. 2010. Development of an integrated methodology for the post-flight analysis of the transition payload on the EXPERT mission. CTR Summer Program Proceedings.

15. Mack, L. M. 1977. Transition and laminar instability. Technical Report JPL PUBLICATION 77-1 5. Pasadena, CA: Jet Propulsion Laboratory, California Institute of Technology.

16. Malik, M. R. 1990. Numerical methods for hypersonic boundary layer stability. J. Comput. Phys. 86(2):376-413.

17. Esfahanian, V. 1999. Computation and stability analysis of laminar flow over a blunt cone in hypersonic flow. Ph.D. Thesis. The Ohio State University. 